\title{
LA EDUCACIÓN CULTURALMENTE RELEVANTE: UN MODELO PEDAGÓGICO PARA LOS ESTUDIANTES DE ORIGEN CULTURAL DIVERSO. CONCEPTO, POSIBILIDADES Y LIMITACIONES
}

\author{
Culturally Relevant Education: A pedagogical model \\ for students from diverse cultural backgrounds. \\ Concept, possibilities and limitations
}

Rosa María RODRÍGUEZ-IZQUIERDO* y Juan Carlos GONZÁLEZ-FARACO*** *Universidad Pablo de Olavide. España.

rmrodizq@upo.es https://orcid.org/0000-0002-9432-1280

*Universidad de Huelva. España.

faraco@ubu.es

bttps://orcid.org/0000-0003-2004-7706

Fecha de recepción: 29/04/2020

Fecha de aceptación: 15/07/2020

Fecha de publicación en línea: 01/011/2020

Cómo citar este artículo: Rodríguez-Izquierdo, R. M. y González-Faraco, J. C. (2021). La educación culturalmente relevante: un modelo pedagógico para los estudiantes de origen cultural diverso. Concepto, posibilidades y limitaciones. Teoría de la Educación. Revista Interuniversitaria, 33(1), 153-172. https://doi.org/10.14201/teri.22990

RESUMEN

Este artículo presenta un estudio de la amplia producción científica sobre la Educación Culturalmente Relevante (ECR), un modelo pedagógico que pretende potenciar significativamente el éxito de los estudiantes culturalmente diversos. Este modelo, de origen norteamericano, es prácticamente desconocido en España. De ahí 
ROSA MARÍA RODRÍGUEZ-IZQUIERDO Y JUAN CARLOS GONZÁLEZ-FARACO

LA EDUCACIÓN CULTURALMENTE RELEVANTE: UN MODELO PEDAGÓGICO PARA LOS ESTUDIANTES DE ORIGEN CULTURAL DIVERSO. CONCEPTO, POSIBILIDADES Y LIMITACIONES

que las aportaciones de este trabajo puedan considerarse una verdadera novedad. Su objetivo es doble: profundizar en el conocimiento de la ECR, cuya eficacia avalan numerosas investigaciones empíricas, y valorar su traslación a nuestro contexto, en el que los alumnos de origen inmigrante suelen tener un rendimiento académico promedio más bajo que el de sus pares. Mediante el análisis de la literatura más importante sobre la ERC, se ha podido: 1, establecer las características teóricas más importantes de este modelo pedagógico, en relación con los de otros enfoques concomitantes y complementarios; 2, estimar su impacto en la mejora del rendimiento del alumnado culturalmente diverso; y 3, determinar sus posibilidades y limitaciones de traslación a nuestro contexto. Los resultados de este trabajo ponen de manifiesto la gran potencialidad de la ECR en la mejora de los procesos de aprendizaje de los estudiantes culturalmente diversos. Por otro lado, se subraya la importancia de la formación inicial y permanente del profesorado en el desarrollo de este modelo educativo, además de sus valiosas orientaciones para la investigación pedagógica y la praxis docente.

Palabras clave: educación culturalmente relevante; educación intercultural; diversidad cultural; pedagogía intercultural; éxito escolar; inclusión social y educativa.

\section{ABSTRACT}

This article presents a study of the extensive scientific production on Culturally Relevant Education (CRE), a pedagogical model that aims to significantly enhance the success of culturally diverse students. This model, of North American origin, is practically unknown in Spain. Hence, the contributions of this work can be considered a true novelty. Its objective is twofold: to deepen knowledge of the CRE, which effectiveness is supported by numerous empirical researches, and to assess its translation to our context, in which students of immigrant origin tend to have lower average academic performance than their peers. Through the analysis of the most important literature on CRE, it has been possible: 1, to establish the most important theoretical characteristics of this pedagogical model, in relation to those of other concomitant and complementary approaches; 2, to estimate its impact on the improvement of the performance of culturally diverse students; and 3, to determine its possibilities and limitations of translation to our context. The results of this work show the great potential of CRE in improving the learning processes of culturally diverse students. Moreover, the importance of initial and ongoing teacher training in the development of this educational model is stressed, in addition to its valuable guidelines for pedagogical research and teaching practice.

Key words: culturally relevant education; intercultural education; cultural diversity; intercultural pedagogy; educational success; social and educational inclusion.

\section{INTRODUCCIÓN, JUSTIFICACIÓN Y MÉTODO}

La preocupación por el fenómeno de la multiculturalidad no es algo nuevo (Gundara, 2014). John Dewey, en Democracia y Educación (1916/1995), ya decía 
que en una ciudad de su tiempo había probablemente más comunidades y tradiciones que en todo un continente en épocas anteriores. Sin embargo, tan asiduo se ha hecho escuchar que vivimos en una sociedad multicultural (Kymlicka, 1996; Kymlicka y Norman, 1999), que este hecho, a pesar de su dimensión y trascendencia, apenas despierta ya la curiosidad en la ciudadanía (Vertovec, 2012). Algunas nociones como «multiculturalidad», «interculturalidad» $\mathrm{y}$ «transculturalidad», tan divulgadas (Jonsen, Maznevski y Schneider, 2011), corren incluso el riesgo de volverse retóricas y vacías de significado. Por si fuera poco, según Han (2018, p. 79), todas ellas coinciden, además, en el error de dar por buena una visión «esencializada» de la cultura y de la identidad cultural, al presuponerlas semánticamente objetivas, definidas, homogéneas y estáticas. Lo inquietante es que, a partir de ese entendimiento, hayan surgido y se hayan legitimado discursos políticos y pedagógicos benéficos y "felices» (Bell y Hartmann, 2007), en cuyo desarrollo, como era previsible, no han tardado en aparecer insuficiencias, disonancias y contradicciones, como, paradigmáticamente, ha sucedido en el contexto educativo (González-Faraco, González-Falcón y Rodríguez-Izquierdo, 2020).

En este enmarañado marco discursivo, este artículo presenta y analiza un modelo pedagógico relativamente nuevo, aunque desconocido en nuestro país, referido a la problemática educativa de las sociedades multiculturales actuales y, específicamente, a las poblaciones escolares culturalmente diversas. Hablamos de la Educación Culturalmente Relevante (en adelante, ECR). No faltan, desde luego, razones que justifiquen la creciente necesidad de un modelo pedagógico como este, a la vista de ciertas tendencias sociales y culturales de la modernidad (Bauman, 2007, 2017; Pardo, 2016), el avance de la globalización económica (Darling-Hammond, 2010; de Sousa Santos, 2006), la expansión de la sociedad digital (Castells, 1997/1998; Han, 2014), la fascinación por el desarrollo de las competencias individuales, y el malestar de una ciudadanía que asiste (a veces atónita, a veces resignada) a la vulneración constante los derechos de los más débiles (Amartya Sen, 2011; Nussbaum, 2015).

En las últimas décadas, al calor de los procesos de globalización y sus múltiples derivaciones, se han intensificado los flujos migratorios en todo el mundo (Sassen, 2010), singularmente hacia Europa. En España, el crecimiento de las cifras ha sido exponencial. Este fenómeno ha alcanzado de lleno, aunque de modo dispar, a todos los ámbitos de la vida social e institucional, con evidente impacto en las escuelas, que se han ido convirtiendo en microcosmos pluriculturales en comunidades cada vez más diversas.

En el caso de España, antes de poner en marcha políticas que podríamos llamar «interculturales», el desafío consistió en responder a una necesidad básica y acuciante: la acogida escolar de la población migrante que iba llegando, a veces en oleadas muy cuantiosas y heterogéneas, y se distribuía muy desigualmente por el territorio nacional. Este proceso, que comenzó a finales del siglo XX, se disparó en la primera década del siglo actual, y representa a estas alturas un fenómeno 
ROSA MARÍA RODRÍGUEZ-IZQUIERDO Y JUAN CARLOS GONZÁLEZ-FARACO LA EDUCACIÓN CULTURALMENTE RELEVANTE: UN MODELO PEDAGÓGICO PARA LOS ESTUDIANTES DE ORIGEN CULTURAL DIVERSO. CONCEPTO, POSIBILIDADES Y LIMITACIONES

sostenido que, en casos, ya ha producido una segunda generación. Con el tiempo, tras ese inicial programa de escolarización de estos alumnos, se inició el tránsito "desde la multiculturalidad (fáctica) hacia la interculturalidad, desde la pluralidad cultural como hecho, al pluralismo como valor». (Bolívar (2007, p. 51). Este tránsito, inconcluso, ha ido configurando toda una pedagogía intercultural genuina (Santos Rego y Lorenzo, 2012), con dos grandes metas: mejorar la calidad de la escolaridad del alumnado de origen extranjero y, en concreto, su rendimiento académico; y favorecer su inclusión social y educativa, en condiciones de igualdad y de ciudadanía compartida. Para alcanzarlas se han puesto en marcha propuestas pedagógicas innovadoras, con especial énfasis en las de carácter lingüístico y las relacionadas con la identidad cultural, y un elenco de iniciativas educativas para la adquisición de competencias interculturales por el conjunto de la comunidad escolar. Sin duda, la participación activa e igualitaria de todo el alumnado, cualquiera que sea su origen, en una sociedad, que se proclama del conocimiento y del aprendizaje, constituye hoy en día un tema central de debate y un reto para los países más desarrollados (OCDE, 2018), si de veras pretenden construir una sociedad más justa y democrática mediante una «educación para todos» (UNESCO, 2000).

Los bajos resultados escolares de los estudiantes migrantes ha sido una cuestión ampliamente analizada en países con una larga historia de inmigración. En ellos se han estudiado y ensayado, a partir de distintas perspectivas teóricas, fórmulas de apoyo a esta población a fin de mejorar sus estándares académicos. En sendos trabajos anteriores (Rodríguez-Izquierdo, 2010a y 2010b), tuvimos ocasión de analizar distintas teorías, enfoques y dispositivos, referidos al éxito escolar de los estudiantes de origen inmigrante y minorías étnicas en los Estados Unidos (EE. UU.). Así como la investigación científica referida a diversos ámbitos de la educación intercultural en España desde los años noventa hasta ahora (Rodríguez-Izquierdo, 2009). Con este, pretendemos profundizar en esta cuestión, concentrando toda la atención en uno de esos enfoques: sin duda, el que ha demostrado un mayor impacto en la mejora del rendimiento de estos estudiantes, la Educación Culturalmente Relevante. La finalidad a la que aspiramos, estudiar y valorar la traslación y aplicabilidad de este modelo a nuestro país, no está exenta de dificultades, dado el exiguo número de investigaciones sistemáticas con que contamos, es obligado acudir a las fuentes originales norteamericanas y, a la postre, estimar su potencial extrapolación a otro contexto.

En efecto, los EE. UU. tienen una dilata trayectoria histórica de acogida de alumnado inmigrante en su sistema educativo, además de una abundante investigación al respecto, con sobradas evidencias científicas que avalan determinadas teorías y enfoques sobre el progreso escolar de este colectivo. En este trabajo, ofrecemos una revisión de la literatura científica en EE. UU. sobre tales modelos, aunque principalmente sobre uno de ellos, la ERC, hasta ahora, como decíamos, poco o nada conocido en España. Nuestra intención es responder, a partir de esa 
revisión, a estas preguntas: ¿Qué enfoques y modelos pedagógicos se han ocupado de la mejora del rendimiento académico de los estudiantes culturalmente diversos y planteado alternativas pedagógicas de interés? Entre ellos, ¿qué características teóricas diferenciales aporta la ECR? ¿Cuáles de sus propuestas podrían ser aplicadas eficazmente al contexto español?

En ningún momento, se ha pretendido llevar a cabo un análisis bibliométrico, en sentido estricto, sobre la producción científica explícitamente dedicada a la ECR, y a otros enfoques y modelos concomitantes y complementarios. A esta información se he ha dado un tratamiento analítico, tratando de dar respuesta a las preguntas antes enunciadas. Lo que, además, permitirá detectar las limitaciones de este enfoque, y sugerir futuras líneas de estudio que ayuden al avance de la investigación sobre el éxito académico de escolares inmigrantes en nuestro contexto.

Es perentorio formular y desarrollar planteamientos realmente inclusivos que eviten, a toda costa, que la escolarización del alumnado culturalmente diverso ${ }^{1}$ se sustancie con la creación de «dispositivos bienintencionados que definen y miden la diferencia, y reconocen su potencial excluyente», pero la encaran con políticas solo formalmente interculturales e inclusivas (González-Faraco et al., 2020, p. 67). En este sentido, hace tiempo que Gibson (1984) denunció un cierto "multiculturalismo benévolo», que, aun proclamando la igualdad de oportunidades de todos los estudiantes con independencia de su origen, concibe el fracaso exclusivamente como resultado de las diferencias culturales. Frente a esta estrecha percepción del problema, Kincheloe y Steinberg (2000), entre otros, ya abrieron en su día nuevos horizontes epistémicos, al incorporar a sus análisis enfoques multiculturales interseccionales, señalando la clase social, el género y la raza como otras variables significativas (Santos Rego et al., 2020). Sin duda, la complejidad multicultural, e hipercultural (Han, 2018), de nuestras instituciones escolares exige una respuesta pedagógica actualizada, de amplias miras y bien fundamentada, para la que una ECR podría significar una opción inspiradora y valiosa.

\section{El MOdELO DE LA EDUCACIÓN CULTURALMENTE RELEVANTE (ECR)}

La ECR aparece en los Estados Unidos en los años noventa del pasado siglo. La teoría que rodea a la ECR está conectada a un cuerpo más amplio de conocimientos sobre la educación multicultural y la ayuda a los estudiantes con bagajes culturalmente diversos que suelen presentar lagunas en el rendimiento académico. En respuesta a estos desafíos, algunos investigadores y profesores creen que la

1. En este trabajo se denomina "estudiantes de origen cultural diverso", en el sentido en que la literatura anglosajona lo entiende (Kymlicka y Norman, 1999), a los estudiantes que difieren de la corriente o grupo principal o mayoritario en términos de procedencia étnica, religiosa, social o lingüística. En el contexto español, mantienen ciertas similitudes con los estudiantes de origen inmigrante que, en buena medida, su propia diversidad los sitúa en un plano de desigualdad. 
ROSA MARÍA RODRÍGUEZ-IZQUIERDO Y JUAN CARLOS GONZÁLEZ-FARACO LA EDUCACIÓN CULTURALMENTE RELEVANTE: UN MODELO PEDAGÓGICO PARA LOS ESTUDIANTES DE ORIGEN CULTURAL DIVERSO. CONCEPTO, POSIBILIDADES Y LIMITACIONES

educación debería adaptarse para aminorar la brecha entre la cultura escolar y la de los hogares. Surge, pues como un enfoque pedagógico que cuestionaba la enseñanza tradicional e invitaba a hacer visibles, respetar y operar con las culturas que los estudiantes traen consigo al sistema escolar (Gay, 2002, 2013, 2018; LadsonBillings, 1995a, 1995b). Hace más de 30 años, que Paris y Ball (2009) consideran la edad de oro de las "pedagogías de los recursos», la ECR plantea una estrategia educativa eficaz para promover el éxito del alumnado de grupos culturales diversos, con un explícito compromiso con la justicia social (Aronson y Laughter, 2016). Pero ¿cuáles son sus rasgos teóricos más distintivos?

En el contexto norteamericano, la extensa investigación sobre la ERC centra su atención en las vías y pautas que ésta propone para dar cabida a las experiencias de los estudiantes de diferentes orígenes culturales en los procesos de enseñanza y aprendizaje. Conviene precisar que, en los EE. UU., la ECR va dirigida comúnmente a sectores de recursos exiguos, principalmente afroamericanos y latinoamericanos. Allí, por tanto, la ERC está vinculada con la economía de la inmigración, las políticas relativas a la adquisición de la lengua, el rendimiento académico y los procesos de aculturación involucrados en todo ello. Ya en 1983, en su informe A Nation at Risk, la Comisión Nacional para la Excelencia en Educación alertaba de las serias deficiencias del sistema educativo norteamericano, y la necesidad de remediarlas. En 2001 la reforma conocida como No Child Left Behind seguía abundando en los mismos objetivos.

La ECR pretendía, desde sus inicios, dar una respuesta a estos problemas desde la perspectiva nueva y propia. Los antecedentes dela ECR se apoyan en las teorías constructivistas al reconocen el valor de los múltiples puntos de vista culturales, pero la ECR también toma préstamos de la pedagogía crítica (Freire, 1998; Giroux, 1984; McLaren,1989), de la pedagogía de la equidad (Banks y Banks, 1995) y de la educación multicultural (Banks y Banks, 2010; Sleeter, 2008). En este trabajo destacamos especialmente el trabajo fundacional de dos investigadoras: Gloria Ladson-Billings (1995a, 1995b, 2014) sobre "pedagogía culturalmente relevante" (culturally relevant pedagogy), y de Geneve Gay (2002, 2013, 2018) sobre «enseñanza culturalmente responsiva" ${ }^{2}$ (culturally responsive teaching). El enfoque de Gay en la enseñanza se centra principalmente en la competencia y los métodos, describiendo lo que un profesor debe hacer en el aula para ser culturalmente sensible. El enfoque de LadsonBillings en la pedagogía busca influenciar actitudes y disposiciones, describiendo la postura que un docente podría adoptar lo que determinaría la planificación, la instrucción y la evaluación. En sus respectivos discursos, ambas suelen recurrir al

2. No es fácil encontrar, en español, un término que traduzca adecuadamente el concepto responsive, Este término en inglés forma parte del campo semántico de la palabra responsibility y alude a la respuesta a algo o a alguien. En este trabajo se aplica a una enseñanza que es "responsiva». Es decir, receptiva y responsable, en el sentido de que tiene en cuenta la diversidad cultural de los estudiantes. 

DE ORIGEN CULTURAL DIVERSO. CONCEPTO, POSIBILIDADES Y LIMITACIONES

término "pedagogía» al someter a discusión la teoría de la enseñanza, y a "enseñanza» para aludir a la práctica educativa. En este artículo, dada su interrelación, se usan los dos, indistintamente.

Ladson-Billings (1995a, p. 18) define la pedagogía culturalmente relevante como "aquella que empodera intelectual, social, emocional y políticamente a los estudiantes mediante el uso en la enseñanza de sus referentes culturales». Y explica, además, cómo y por qué las creencias del profesorado, su sentido ético y sus ideas sobre la docencia, son factores sustantivos en la acogida que dispensan a los estudiantes culturalmente diversos.

Gay (2018, p. 111), en una dirección similar, sugiere que «el objetivo fundamental de la enseñanza culturalmente responsiva es capacitar a los estudiantes de diferentes grupos étnicos y culturales para el éxito académico, la afiliación cultural y la eficacia personal». Según esta autora, los principios teóricos de este enfoque se basan, primeramente, en la idea de que la individualidad de los estudiantes está profundamente "entrelazada con su identidad étnica y su socialización cultural» (Gay, 2018, p. 25). Por ello, aunque la cultura viene a ser un ensamblaje de variables (tales como género, edad, estatus socioeconómico, lengua, religión, etc.), la identidad racial y étnica resulta ser un factor dominante en la identidad cultural, al darse una "consistencia de patrones de desempeño entre los grupos étnicos» (Gay, 2018, p. xvii). En segundo lugar, los alumnos solo pueden ser «mejores personas» y "mejores estudiantes" si los docentes tienen en cuenta sus referentes culturales en la enseñanza. Por desgracia, es usual que el profesorado apenas esté familiarizado con las culturas de origen de su alumnado, lo que limita su capacidad de comprensión y puede hacerlo proclive a prejuicios y estereotipos, además de influir en su pensamiento didáctico y en su ejercicio profesional (González-Faraco, Jiménez y Pérez-Moreno, 2013; Hollie, 2018; Villegas y Lucas, 2002; Rodríguez-Izquierdo, González Falcón y Permisán, 2020; Sleeter, 2008).

Gay (2018, p. 24) asegura que la «enseñanza culturalmente responsiva» es un "paradigma pedagógico muy diferente» que reconoce la singularidad cultural de cada estudiante y, en consecuencia, se vale "del conocimiento de sus experiencias previas y de sus marcos culturales de referencia para hacer el aprendizaje más relevante y eficaz. Se enseña los y a través de los puntos fuertes de los estudiantes, validándolos y afirmándolos». Según este enfoque, los estudiantes «deben convertirse en estudiosos de la diversidad cultural y generar su propio contenido en el currículum» (Gay, 2018, p. 144), en el cuadro de una enseñanza que potencie no solo la dimensión académica sino también la emocional y psicológica. Por supuesto, además de "culturalmente relevante» (Ladson-Billings, 1995a; 1995b), este modelo educativo y/o pedagógico ha continuado desarrollándose y ha recibido otras denominaciones, tales como "pedagogía culturalmente apropiada» (Au, 2007), "culturalmente congruente" (Mohatt y Erickson, 1981), "culturalmente responsable» (Erickson y Mohatt, 1982), o más recientemente «educación relevante» (Aronson y Laughter, 2016) y "culturalmente sostenible» (Paris y Alim, 2017). Últimamente, se 

DE ORIGEN CULTURAL DIVERSO. CONCEPTO, POSIBILIDADES Y LIMITACIONES

pone el énfasis en que, sea cual sea su nombre, la ECR exige un cambio sustancial de perspectiva, lo que implica repensar radicalmente las prácticas de enseñanza (Gay, 2002, 2013, 2018; Hollie, 2018).

Algunos trabajos (Aronson y Laughter, 2016; Au, 2007; Dover, 2013), en vez de resaltar las diferencias - a veces, meros matices- entre las distintas formulaciones de este enfoque, prefieren destacar sus coincidencias: 1) el objetivo de la ECR es aumentar el éxito educativo de estudiantes culturalmente diversos; 2) este éxito se logrará mediante la construcción de puentes en el sistema familia-escuela; 3) el objetivo es mejorar (o al menos preservar) la competencia de los estudiantes en su cultura e idioma nativos; y 4) es fundamental promover la justicia social, la igualdad y la celebración de la diversidad.

Para Richards, Brown y Forde (2006), la ECR abarca tres vertientes que interactúan, de ahí su complejidad, en el proceso de enseñanza-aprendizaje: la institucional (políticas y valores); la personal (aspectos cognitivos y emocionales de los profesores y su receptividad a la diversidad cultural) y la educativa (estrategias, materiales y actividades de enseñanza). En sintonía con la teoría sociocultural, la ECR entiende, y este es uno de sus principios fundamentales, que la enseñanza es una práctica mediada culturalmente que tiene lugar en un determinado ámbito social, y que, por consiguiente, debe ser contextualizada (Rodríguez-Izquierdo, 2011b). Lo que la ECR añade es que no puede haber aprendizaje significativo al margen de un contexto cultural concreto. Desde una perspectiva vygotskiana, la ECR parte, por un lado, de los conceptos que los estudiantes aprenden de manera espontánea a través de sus experiencias informales y, por otro, del uso que los docentes hacen del patrimonio cultural de los estudiantes en el proceso de construcción de nuevos aprendizajes. En otras palabras, se trata de una enseñanza ligada a la cultura (Dover, 2013), pues es claro que ésta desempeña un papel crítico en la comunicación y en la recepción de información, así como en la configuración de los procesos cognitivos (Lee, 2007).

Es importante insistir en el papel crucial que juegan los profesores en la ECR. Que éstos reconozcan la cultura, única y valiosa, de cada estudiante y se valgan de sus experiencias vitales como recursos pedagógicos, puede contribuir a que la enseñanza sea más accesible y equitativa para todos (Andrews, 2017; Villegas y Lucas, 2002). Nieto (2002), Sleeter (2008) y Rodríguez-Izquierdo, González Falcón y Permisán (2020), entre otros, demuestran que las creencias de los docentes determinan el sentido y el enfoque de sus prácticas de enseñanza. Hay profesores, con una "perspectiva intercultural", que piensan que los estudiantes aportan una gran cantidad de conocimientos y una herencia lingüística propia que pueden enriquecer el currículum. Hay, en cambio, otros, alineados con la "perspectiva del déficit", que piensan en los estudiantes culturalmente diversos como ignorantes a los que hay que adiestrar y en cierto modo "redimir» de su "déficit» cultural. En manos de estos docentes, a juicio de estas autoras, las estrategias pedagógicas más avanzadas se vuelven ineficaces. 
En el polo opuesto a la perspectiva del déficit, la ECR concibe al otro como un sujeto activo, con cultura e identidad propia, y derecho a contribuir a la construcción de sociedades democráticas; y no como ente pasivo que debe ser asimilado por la cultura dominante o globalizadora. Siguiendo esa lógica, la escuela, contemplada como comunidad cultural plural, será la que deba adaptarse al alumnado y no a la inversa. La ECR apunta, más allá de los meros resultados académicos, hacia la meta más comprensiva de la mejora educativa, en términos de acceso igualitario de todos a una enseñanza de calidad y a la participación en la vida ciudadana (Banks, 2009). En este sentido, la ECR podría ser concebida como una vía óptima para desarrollar la que Walsh $(2009,2010)$ llama "interculturalidad crítica", un discurso que se adentra en la genealogía y significado político de la diferencia cultural. Y pone el énfasis en la educación como proyecto político que alienta el reconocimiento de la diversidad de saberes (de Sousa, 2017), y la defensa de los derechos humanos. Puede, de este modo, colaborar en la construcción de sociedades que asuman la diferencia como constitutiva de la democracia, y empoderen a aquellos grupos sociales y culturales que han sido históricamente minusvalorados y damnificados (Candau, 2013). En este marco, la ECR se afana en fomentar el pluralismo lingüístico y cultural como parte del proyecto democrático de la escolarización.

Se han realizado muchas experiencias exitosas derivadas de este enfoque incorporando los principios y el uso de las estrategias de ECR dentro del aula, entre las que destaca el libro Culturally responsive methodologies (Berryman, SooHoo y Nevin, 2013). Las respuestas incluidas en esta publicación son prueba de que se trata de una forma positiva y efectiva de pedagogía.

\section{LOS BENEFICIOS DE UNA ECR PARA EL ALUMNADO DE ORIGEN CULTURAL DIVERSO}

Durante las décadas de los años sesenta y setenta, la investigación social trató de explicar las diferencias de rendimiento de los estudiantes de origen cultural diverso, ateniéndose a la óptica del déficit, es decir, catalogándolos como un grupo "distinto" que sufre desventajas sociales y escolares, que deberían ser suplidas mediante programas específicos de cariz terapéutico (González-Faraco et al., 2020; Valencia, 2010; Talavera y Guzmán, 2018).

Desde el siglo XIX, en los EE. UU., los descendientes de los antiguos esclavos negros, los asiáticos y los nativos americanos, así como algunos grupos europeos, fueron considerados como «los otros» y por tanto «no civilizados». Tradicionalmente, estos grupos, si bien unos más que otros, han sufrido marginación en las escuelas, por tratarse, aparentemente, de alumnos «atrasados» (Popkewitz, 2002), quienes, para recuperarse, debían sumir su identidad cultural en un supuesto melting pot nacional.

Sin embargo, la investigación disponible, en abierta contradicción con esa premisa, demuestra con datos que los estudiantes con una fuerte identificación étnica tienden a conseguir buenos aprendizajes. Así, por ejemplo, un estudio de Altschul, Oyserman y Bybee (2008) con 185 estudiantes latinos indica que los que 

DE ORIGEN CULTURAL DIVERSO. CONCEPTO, POSIBILIDADES Y LIMITACIONES

alcanzan mejores resultados son los que se identifican tanto con su origen étnico como con la cultura de acogida. Por contra, aquellos que se identifican poco con su cultura o exclusivamente con ella, y en absoluto con la de la sociedad de acogida, suelen tener escaso éxito. Este y otros trabajos similares subrayan la importancia de reconocer y trabajar con las culturas de los estudiantes y, asimismo, el error de asimilarlos a la cultura de acogida.

Por otra parte, la investigación más reciente también constata que el bajo rendimiento académico del alumnado culturalmente diverso guarda relación con las discontinuidades culturales entre familia y escuela (Rodríguez-Izquierdo, 2010a, 2010b). Es decir, con el desajuste entre los procesos de socialización primaria y las prácticas escolares, cuando éstas se abstraen de los marcos de las referencias y códigos culturales de estos estudiantes (Gay, 2018; Nieto, 2002; Rodríguez-Izquierdo, 2011a). Investigadoras como Irvine (2003) y Hollie (2018) aportan evidencias de que cuando hay continuidad familia-escuela, la enseñanza es más eficaz y es menos probable que los docentes opten por el enfoque del déficit cultural.

Ya el antropólogo John Ogbu (1982) había sugerido que todos los estudiantes experimentan este tipo de discontinuidades a lo largo de su escolaridad. Bourdieu, por su parte, explicó con claridad que la cultura simbólica de la escuela responde más a los códigos de cultura burguesa que a los de la clase trabajadora, o sea, a su diferencia de capital cultural, lo que explicaría el origen social del éxito o el fracaso y la consiguiente reproducción escolar de las desigualdades. Según otros autores (Gay, 2018; Ladson-Billings, 1995a y 1995b; Suárez-Orozco y Todorova, 2008), las mayores discordancias se observan precisamente en los estudiantes pertenecientes a minorías lingüísticas y culturales. Éstos suelen encontrar más barreras para atribuir sentido y significado a los contenidos escolares, a causa de esa discontinuidad cultural familia-escuela, lo que termina condicionando su rendimiento. Gay (2018, p. 46) lo argumenta de este modo:

La mayoría de los docentes esperamos que los estudiantes se comporten de acuerdo con los estándares de la cultura escolar. Cuando los estudiantes no lo logran, los profesores los consideran problemáticos y difíciles y, en lugar de construir sobre lo que los estudiantes traen para hacer su aprendizaje mejor y más fácil, los profesores quieren corregir y compensar sus déficits culturales. Esto significa que los estudiantes culturalmente diversos tienen que ajustarse a las normas culturales eurocéntricas y de clase media.

Efectivamente, cuando el conocimiento académico y las competencias que se deben adquirir en el aula se corresponden con las experiencias vitales y los marcos culturales de los estudiantes, éstos muestran más interés, se sienten más comprometidos y aprenden con mayor profundidad (Ladson-Billings, 1995a; Nieto, 2002). Gay (2018) y Villegas y Lucas (2002), por su parte, insisten en que la conservación de los valores étnicos, el bilingüismo y la participación en la comunidad favorecen el éxito educativo. 
Por otra parte, la ECR reitera que el rendimiento mejora cuando los docentes logran establecer conexiones significativas entre sus acciones pedagógicas y los conocimientos y prácticas culturales de su alumnado (Ladson-Billings, 1995a; Lee, 2007). Cuando el profesorado cobra conciencia de esta relación entre enseñanza y cultura comienza a servirse de los llamados funds of knowledge de sus estudiantes que son «los conocimientos y habilidades esenciales para el funcionamiento colectivo e individual y el bienestar, acumulados históricamente». (González, Moll y Amanti, 2005, p. 133). Todos los alumnos disponen de recursos (o fondos del conocimiento) a los que sus profesores pueden acudir para incentivar sus aprendizajes. EstebanGuitart y Saubich (2013) ofrecen un ilustrativo ejemplo de trabajo con estos fondos como estrategia para conciliar la práctica escolar y la cultura familiar. En otro estudio, Esteban-Guitart y Vila (2013) se interesan por el provechoso cambio de perspectiva pedagógica que tal conciliación representa. En un reciente texto, Lorenzo, Míguez y Cernadas (2020) estudian la idoneidad de los fondos de conocimiento de las familias gitanas, como fuente para el diseño de propuestas de educación inclusiva que estimulen su implicación en la formación de sus hijos, lo que acrecentaría sus posibilidades de progreso escolar.

Otras investigaciones también han constatado altos niveles de participación de los estudiantes culturalmente diversos, cuando se utilizan recursos relevantes para su vida. Por ejemplo, Copenhaver (2001) analizó cómo estudiantes afroamericanos respondían a la lectura, en pequeño grupo, del libro Malcolm X: A Fire Burning Brightly. Y comprobó que tenían bastante más conocimiento de la vida de Malcolm $\mathrm{X}$ que el que sus profesores inicialmente imaginaban. Los estudiantes, altamente motivados, usaron resueltamente el conocimiento adquirido a través de los medios afroamericanos, los líderes de los derechos civiles y las cuestiones raciales de su vida cotidiana, para seguir la trama de la lectura, descubrir relaciones e interpretar la historia de la obra. Se han observado y documentado numerosos efectos positivos del programa de fondos de conocimiento, que convergen con los objetivos de una ECR. Podríamos sintetizarlos en cuatro enunciados: 1) modifican las creencias del profesorado en torno a las familias de grupos culturales diversos; 2) crean nuevas relaciones entre docentes y familias basadas en la confianza mutua y la reciprocidad; 3) fomentan innovaciones didácticas mediante unidades curriculares que parten de los fondos de conocimiento del alumnado; y 4) consiguen una actitud más favorable hacia el aprendizaje, con la consecuente mejora de su rendimiento (González, Moll y Amanti, 2005).

\section{LIMITACIONES DE LA ECR}

No faltan voces críticas que plantean algunas objeciones a la ERC y advierten de sus limitaciones teóricas y prácticas. La primera de ellas alude al acento que la ECR pone en la cultura, lo que, para algunos autores, podría ir en detrimento del logro de las competencias académicas básicas de los estudiantes, como la lectoescritora, 
ROSA MARÍA RODRÍGUEZ-IZQUIERDO Y JUAN CARLOS GONZÁLEZ-FARACO LA EDUCACIÓN CULTURALMENTE RELEVANTE: UN MODELO PEDAGÓGICO PARA LOS ESTUDIANTES DE ORIGEN CULTURAL DIVERSO. CONCEPTO, POSIBILIDADES Y LIMITACIONES

la científica y la matemática. Y llegan a achacarle una falta de profundidad y rigor científico. A este respecto, trabajos como los de Brown y Crippen (2017) y Brown (2017) ponen de relieve que los profesores tienden a oponer ciencia y cultura, acaso porque, abusando de una perspectiva positivista, aplican un sentido fundamentalmente disciplinar al currículum.

Disponemos, sin embargo, de numerosas investigaciones en el área de las matemáticas (la materia en la que muchos de estos estudiantes obtienen peores resultados), cuyas conclusiones dan cuenta de las ventajas de una ECR para mitigar ese problema. Lipka y otros (2005) lo han analizado mediante un estudio experimental que evalúa el aprendizaje de los alumnos en relación con los estándares y las pruebas estatales de rendimiento, y verifican que los que habían seguido el plan ${ }^{3}$ de estudios de Math in a Cultural Context sacaban mejores calificaciones que los que no. Nasir (2000), en la misma línea, estudió la relación entre la construcción de la identidad cultural y el aprendizaje de las matemáticas, y encontró que esta relación es un factor sustancial para el éxito en esta disciplina de los adolescentes afroamericanos. Basándose en el conocimiento que estos tienen del juego del dominó, esta investigadora llegó a la conclusión de que, en las actividades en las que se trabajaban los conceptos matemáticos acercándolos a su experiencia cotidiana, eran capaces de resolver los problemas y alcanzar los objetivos propuestos. Martin (2000), tras evaluar también el nivel de competencia matemática de los estudiantes afroamericanos, llegaba a deducciones similares, y sugería a los profesores que desarrollaran la conciencia cultural de estos alumnos en torno a todo aquello que influye en su vida escolar. Civil y Khan (2001) investigaron a docentes que recurrían a las experiencias de los estudiantes de origen cultural diverso, y en concreto a la plantación de jardines, para el aprendizaje de conceptos matemáticos importantes. Ensign (2003), por su parte, estudió cómo una maestra utilizaba la comparación de precios en las tiendas del barrio como medio para conseguir una mejor comprensión de conceptos matemáticos abstractos.

Una segunda objeción pone en entredicho el carácter conceptualmente novedoso o transformador de la ERC. Irvine y Armento (2000) sostienen que, en realidad, este enfoque educativo ha formado parte de la vida de las escuelas de los EE. UU. desde su época fundacional, si bien solo se destinaba a los alumnos pertenecientes a la cultura mayoritaria, es decir, a los blancos nacidos en el país, de clase media y habla inglesa. Efectivamente, la educación americana se ha desenvuelto históricamente en un medio cultural en el que se ha postergado, en general, a los estudiantes de origen cultural diverso, cuyos sus resultados han sido normalmente peores que los de los estudiantes blancos. En esencia, Irvine y Armento sostienen que la cultura escolar norteamericana ha estado profundamente impregnada por una cosmovisión

3. Se trata de un proyecto curricular que conecta la cultura y el conocimiento cultural de los Yup'ik con las matemáticas. 
eurocéntrica y, por tanto, por una ideología que omitía o menoscababa la historia, las vivencias, la cultura, en suma, de los estudiantes de minorías culturales, los pobres y las mujeres.

La tercera de las objeciones acusa a la ECR de que solo resulta idónea para grupos étnicos minoritarios y sectores sociales vulnerables o en riesgo de exclusión, y que, por lo tanto, tiene una funcionalidad restringida, e incluso podría representar una vía de discriminación. No son pocos los estudios que cuestionan la idea de proporcionar un apoyo específico a ciertos grupos. Moon (2011), de hecho, avisa de la estigmatización que puede derivarse de destinar este tipo de modelo pedagógico a los grupos culturalmente diversos, teniendo en cuenta la creencia, tan extendida, de que las diferencias culturales son las «anclas fundamentales de todos los demás comportamientos». (Gay, 2018, p. 11), lo que se suele traducir en una concepción estereotipada de la diversidad cultural. Para Moon, una de las voces más críticas al respecto, el discurso convencional de la ECR es muy discutible, porque parte de la naturalización de la cultura y la identidad cultural. A su parecer, la ERC presupone que cada grupo cultural tiene un patrón de rendimiento propio y distinto al de otros grupos.

En relación con esta última objeción, Ladson-Billings (1995b) ya había aclarado que la ECR no es, de ningún modo, una pedagogía específica para grupos especiales, sino un modelo potencialmente bueno para todos. En aulas "culturalmente relevantes", cuentan las aportaciones de todos los estudiantes para promover una formación de calidad al servicio de todos. Gay (2000), sin embargo, marca distancias, al menos en parte, con esta posición tan optimista. A su juicio, y al de otros muchos, la idea de que hay una educación "universalmente buena» olvida la influencia que la clase, la raza, el género, la etnia o la cultura de estudiantes y maestros ejercen en los procesos educativos, y que los estándares de excelencia en la escuela están filtrados culturalmente y favorecen las desigualdades.

Finalmente, y ésta sería una cuarta objeción a la ERC, Moon (2011) afirma que el simple aumento de la competencia cultural de los docentes o el desarrollo de currículos culturalmente relevantes, no alteran, sustancialmente las reglas por las que se clasifica y se representa a los estudiantes de origen cultural diverso. En su opinión, la noción convencional de la ECR ignora cómo actúa el poder a través de actitudes estereotipadas y comparaciones entre el alumnado de culturas minoritarias y el de la cultura dominante, mediante escalas implícitas y asiduamente arbitrarias. En ese sentido, la ERC no representaría un giro epistemológico radical, puesto que omite los factores sociopolíticos estructurales. Ciertamente, no es suficiente ser sensible a los valores culturales de los estudiantes culturalmente diversos. La ERC debiera, por ello, ensanchar su campo de acción (Paris y Alim, 2017), y animar a los educadores a que trabajen simultáneamente en dos frentes complementarios (Au, 2007): ayudar a los estudiantes a alcanzar estándares educativos más altos y competir con éxito en la economía global, a la par que esforzarse en preservar sus culturas y lenguas nativas. 
ROSA MARÍA RODRÍGUEZ-IZQUIERDO Y JUAN CARLOS GONZÁLEZ-FARACO LA EDUCACIÓN CULTURALMENTE RELEVANTE: UN MODELO PEDAGÓGICO PARA LOS ESTUDIANTES DE ORIGEN CULTURAL DIVERSO. CONCEPTO, POSIBILIDADES Y LIMITACIONES

\section{CONSIDERACIONES FINALES}

En la literatura norteamericana se dispone de un nutrido cuerpo de investigaciones que aportan datos empíricos sobre los fundamentos teóricos de la ECR y sus beneficios educativos. También, como acabamos de observar, sugieren una serie de debilidades en las que valdría la pena profundizar.

La ECR, y acaso ésta sea su idea fundamental, pone de manifiesto que la presencia de estudiantes de culturas diversas en el medio escolar exige un nuevo planteamiento pedagógico y ético, según el cual la diferencia cultural del otro debe transformarse en deferencia hacia el otro. Multitud de estudios muestran con reiteración que la aplicación de la ECR influye positivamente en el compromiso de los estudiantes de origen cultural diverso con la enseñanza (Gay, 2018), y que sus posibilidades de éxito escolar crecen cuando el profesorado se decanta por una enseñanza culturalmente relevante (Gay, 2018; Ladson-Billings, 1995a, 1995b), se siente concernido por el desarrollo integral de sus estudiantes y comprende las implicaciones culturales, éticas y políticas de su tarea. Cuando así sucede, los estudiantes se benefician de un mejor aprendizaje a la vez que conservan su identidad cultural: se convierten en sujetos biculturales en el marco de una nueva cultura. La ECR no es otro método más, sino una forma distinta de pensar acerca de este alumnado, al que ofrece alternativas pedagógicas eficientes. En consecuencia, pone en cuestión los enfoques de la enseñanza basados en la teoría del déficit, que es, al menos en parte, responsable de los bajos resultados del alumnado culturalmente diverso (Valencia, 2010). Trabajos como los de Irvine (2003), Lee (2007) y Moll (2000) juzgan severamente esta teoría que considera a estos estudiantes como "deficientes», siendo tan solo "diferentes».

La ECR propone la ruptura con una visión etnocéntrica de la enseñanza, y la invita a abrirse a los contenidos culturales que aportan los propios estudiantes (González; Moll y Amanti, 2005; Moll, 2000). No se pretende con ello sobrealimentar el currículum con nuevos contenidos, sino hacerlo permeable a la diversidad cultural de la comunidad educativa, a sabiendas de que la enseñanza es una actividad mediada por la cultura (Rodríguez-Izquierdo, 2011). El reto, en todo caso, es concretar los principios de la ECR en la práctica pedagógica. Para ello, ofrece un elenco de principios y pautas que pueden ser de gran utilidad para guiar la praxis educativa intercultural en pro de una mejora general de la calidad de la enseñanza. Para ello, sería de gran interés la recopilación exhaustiva de referencias documentadas de proyectos y experiencias, es decir, de buenas prácticas educativas congruentes con el enfoque de la ERC.

En un contexto crecientemente multicultural se va a requerir un profesorado más formado y comprometido (Irvine, 2003; Nieto, 2002), que construya significados positivos de la diversidad cultural, distanciándose de modelos basados en el déficit cultural o de políticas terapéuticas o compensadoras. Darling-Hammond (1997, p. 295) subraya la trascendencia educativa de que los profesores tengan en cuenta «las 
diferencias culturales, el idioma, la familia, la comunidad, el género, la escolaridad previa y otros factores que moldean la experiencia de las estudiantes». Este conocimiento contribuirá a que logren una mejor comprensión de la riqueza de recursos culturales que los estudiantes traen al aula, y a ser capaces de involucrarlos en prácticas dialógicas en las que conceptos como diferencia cultural, discriminación y poder se hagan presentes (Nieto, 1999; Sleeter, 2008, 2011).

Acaso quede mucho camino hasta conseguir que los docentes reciban una sólida formación inicial y permanente sobre este modelo, lo que podría suponer un paso crítico para responder a las particularidades y necesidades de los estudiantes de origen inmigrante. Este estudio proporciona algunas orientaciones, creemos que valiosas, para formular políticas y prácticas educativas destinadas a una enseñanza culturalmente relevante.

\section{REFERENCIAS BIBLIOGRÁFICAS}

Altschul, I., Oyserman, D. \& Bybee, D. (2008). Racial-ethnic self-schemas and segmented assimilation: Identity and the academic achievement of Hispanic youth. Social Psychology Quarterly, 71(3), 302-320. https://doi.org/10.1177/019027250807100309

Andrews, K. (2017). Culture, Curriculum, and Identity in Education. [Book Review]. Journal of Ethnic and Cultural Studies, 4(2), 99-101. https://doi.org/10.1057/9780230105669_1

Aronson, B. \& Laughter, J. (2016). The theory and practice of culturally relevant education: A synthesis of research across content areas. Review of Educational Research, 86(1), 163-206. https://doi.org/10.3102/0034654315582066

Au, K. H. (2007). Culturally responsive instruction: Application to multiethnic classrooms. Pedagogies: An International Journal, 2(1), 1-18. https://doi.org/10.1080/15544800701343562

Banks, J. A. (2009). Diversity and citizenship education in multicultural societies. Multicultural Education Review, 1(1), 1-28. https://doi.org/10.1080/23770031.2009.11102861

Banks, C. A. \& Banks, J. A. (1995) Equity pedagogy: An essential component of multicultural education. Theory into Practice, 34(3), 152-158. https://doi.org/10.1080/00405849509543674

Banks, J. A. \& Banks, C. A. (Eds.) (2010). Multicultural education: Issues and perspectives ( $7^{\mathrm{a}}$ ed.). John Wiley.

Bauman, Z. (2007). Los retos de la educación en la modernidad líquida. Gedisa.

Bauman, Z. (2017). La cultura en la modernidad líquida. FCE.

Bell, J. M.\& Hartmann, D. (2007). Diversity in everyday discourse: The cultural ambiguities and consequences of 'happy talk'. American Sociological Review, 72(6), 895-914. https:// doi.org/10.1177/000312240707200603

Berryman, M., Soo Hoo, S., \& Nevin, A. (Eds.). (2013). Culturally responsive methodologies. Emerald Group Publishing.

Bolívar, A. (2007). Educación para la ciudadanía Algo más que una asignatura. Graó.

Brown, J. C. (2017). A metasynthesis of the complementarity of culturally responsive and inquiry-based science education in K-12 settings: Implications for advancing equitable science teaching and learning. Journal of Research in Science Teaching, 54(9), 1143-1173. https://doi.org/10.1002/tea.21401 
ROSA MARÍA RODRÍGUEZ-IZQUIERDO Y JUAN CARLOS GONZÁLEZ-FARACO LA EDUCACIÓN CULTURALMENTE RELEVANTE: UN MODELO PEDAGÓGICO PARA LOS ESTUDIANTES DE ORIGEN CULTURAL DIVERSO. CONCEPTO, POSIBILIDADES Y LIMITACIONES

Brown, J. C. y Crippen, K. J. (2017). The knowledge and practices of high school science teachers in pursuit of cultural responsiveness. Science Education, 101(1), 99-133. https:// doi.org/10.1002/sce. 21250

Candau, V. M. (2013). Educación intercultural crítica: construyendo caminos. En C. Walsh (Ed.), Pedagogías decoloniales: prácticas insurgentes de resistir, (re)existir y (re)vivir (pp. 69-103). Abya-Yala.

Castells, M. (1997/1998). La era de la información: economía, sociedad y cultura. Alianza, 3 vols.

Civil, M. \& Khan, L. (2001). Mathematics instruction developed from a garden theme. Teaching Children Mathematics, 7(7), 400-405.

Copenhaver, J. (2001). Listening to their voices connects literary and cultural under- standings: Responses to small group read-alouds of Malcolm X: A Fire. New Advocate, 14, 343-359.

Darling-Hammond, L. (2010). The flat world and education. Teachers College Press.

Dewey, J. (1916/1995). Democracia y educación. Morata.

Dover, A. G. (2013). Teaching for social justice: From conceptual frameworks to classroom practices. Multicultural Perspectives, 15(1), 3-11. https://doi.org/10.1080/15210960.20 13.754285

Erickson, F. \& Mohatt, G. (1982). Cultural organization and participation structures in two classrooms of Indian students. En G. Spindler (Ed.), Doing the ethnography of schooling (pp. 131-174). Holt, Rinehart \& Winston.

Ensign, J. (2003). Including culturally relevant math in an urban school. Educational Studies, 34(4), 414-423.

Esteban-Guitart, M. y Vila, I. (2013). La modificación de las creencias sobre las familias de origen inmigrante por parte de docentes después de participar en el proyecto educativo fondos de conocimiento. Cultura y Educación, 25(2), 241-254. https://doi. org/10.1174/113564013806631282

Esteban-Guitart, M. y Saubich, X. (2013). La práctica educativa desde la perspectiva de los fondos de conocimiento e identidad. Teoría de la Educación. Revista Interuniversitaria, 25(2), 189-211. https://doi.org/10.14201/11583

Freire, P. (1998). Pedagogy of the oppressed. Continuum.

Gay, G. (2002). Preparing for culturally responsive teaching. Journal of Teacher Education, 53, 106-116.

Gay, G. (2013). Teaching to and through cultural diversity. Curriculum Inquiry, 43(1), 48-70. https://doi.org/10.1111/curi.12002

Gay, G. (2018). Culturally responsive teaching. Theory, research, and practice. Teachers College Press. (3. ${ }^{\mathrm{a}}$ ed.).

Gibson, M. A. (1984). Approaches to multicultural education in the United States: Some concepts and assumptions. Anthropology \& Education Quarterly, 15(1), 94-119.

Giroux, H. A. (1984). Ideology, culture, and the process of schooling. Falmer Press.

González, N., Moll, L. \& Amanti, C. (Eds.). (2005). Funds of knowledge: Theorizing practices in housebolds, communities, and classrooms. Routledge.

González-Faraco, J. C., Jiménez, J. R. \& Pérez-Moreno, H. M. (2013). Bitter strawberries: the construction of differences in a multicultural school: a case study. Intercultural Education, 24(6), 592-604. 
ROSA MARÍA RODRÍGUEZ-IZQUIERDO Y JUAN CARLOS GONZÁLEZ-FARACO

LA EDUCACIÓN CULTURALMENTE RELEVANTE: UN MODELO PEDAGÓGICO PARA LOS ESTUDIANTES DE ORIGEN CULTURAL DIVERSO. CONCEPTO, POSIBILIDADES Y LIMITACIONES

González-Faraco, J. C., González-Falcón, I. y Rodríguez-Izquierdo, R. (2020). Políticas interculturales en la escuela: significados, disonancias y paradojas. Revista de Educación, 387, 67-88. https://doi.org/10.4438/1988-592X-RE-2020-387-438

Gundara, J. (2014). Global and civilisational knowledge: Eurocentrism, intercultural education and civic engagements. Intercultural Education, 25(2), 114-127. https://doi.org/10.10 80/14675986.2014.888802

Han, B.-Ch. (2014). En el enjambre. Herder.

Han, B.-Ch. (2018). Hiperculturalidad. Herder.

Hollie, S. (2018). Culturally and linguistically responsive teaching and learning ( ${ }^{\mathrm{a}}$ ed.). Huntington Beach, CA: Shell Educational.

Irvine, J. \& Armento, B. (Eds.). (2000). Culturally responsive teaching. Lesson planning for the elementary and middle grades. McGraw Hill.

Irvine, J. (2003). Educating teachers for a diverse society: Seeing with the cultural eye. Teachers College Press.

Jonsen, K., Maznevski, M. L. \& Schneider, S. (2011). Diversity and it's not so diverse literature: An international perspective. International Journal of Cross Management, 11(1), 35-62. https://doi.org/10.1177/1470595811398798

Kincheloe, J. y Steinberg, S. (2000). Repensar el multiculturalismo. Octaedro.

Kymlicka, W. (1996). Ciudadanía multicultural. Una teoría liberal de los Derechos Humanos de las minorías. Paidós.

Kymlicka, W. \& Norman, W. (Eds.). (1999). Citizenship in diverse societies. Oxford University Press.

Ladson-Billings, G. (1995a).Toward a theory of culturally relevant pedagogy. American Educational Research Journal, 32, 465-491.

Ladson-Billings, G. (1995b). But that's just good teaching: The case for culturally relevant pedagogy. Theory into Practice, 34, 159-165. https://doi.org/10.1080/00405849509543675

Lee, C. (2007). Culture, literacy, and learning. Teachers College Press.

Lipka, J., Hogan, M. P., Parker Webster, J., Yanez, E., Adams, B., Clark, S. \& Lacy, D. (2005). Math in a cultural context: Two case studies of a successful culturally- based math project. Anthropology E Education Quarterly, 36(4), 367-385. https://doi.org/10.1525/ aeq.2005.36.4.367

Lorenzo, M., Míguez, G. y Cernadas, F. (2020). ¿Pueden contribuir los fondos de conocimiento a la participación de las familias gitanas en la escuela? Bases para un proyecto educativo. Teoría de la Educación. Revista Interuniversitaria, 32(1), 191-211. https:// doi.org/10.14201/teri.21299

Martin, D. (2000). Mathematics success and failure among African American youth: The role of sociohistorical context, community forces and school influences $\xi$ influence and individual agency. Lawrence Erlbaum.

McLaren, P. (1989). Life in schools: An introduction to critical pedagogy in the foundations of education. Longman.

Mohatt, G. \& Erickson, F. (1981). Cultural differences in teaching styles in an Odawa school: A sociolinguistic approach. En H. Trueba, G. Guthrie \& K. Au (Eds.), Culture and the bilingual classroom: Studies in classroom ethnography (pp. 105-119). Newbury House. 
ROSA MARÍA RODRÍGUEZ-IZQUIERDO Y JUAN CARLOS GONZÁLEZ-FARACO LA EDUCACIÓN CULTURALMENTE RELEVANTE: UN MODELO PEDAGÓGICO PARA LOS ESTUDIANTES DE ORIGEN CULTURAL DIVERSO. CONCEPTO, POSIBILIDADES Y LIMITACIONES

Moll, L. (2000). Inspired by Vygotsky: Ethnographic experiments in education. En C. Lee \& P. Smagorinsky (Eds.), Vygotskian perspectives on literacy research: Constructing meaning through collaborative inquiry (pp. 256-268). Cambridge University Press.

Moon, S. (2011). Rethinking culturally responsive teaching: Toward new (im)possibilities of curriculum studies and policy. Multicultural Education Review, 3(2), 69-102. https://doi. org/10.1080/23770031.2009.11102884

Nasir, N. (2000). Points ain't everything: Emergent goals and average, and percent understanding in the play of basketball among African American students. Anthropology $\mathcal{E}$ Educational Quarterly, 31, 283-305. https://doi.org/10.1525/aeq.2000.31.3.283

Nieto, S. (1999). The light in their eyes: Creating multicultural learning communities. Teachers College Press.

Nieto, S. (2002). Language, culture, and teaching: Critical perspectives for a new century. Lawrence Erlbaum.

No Child Left Behind (NCLB). (2003). Act of 2001. https://www2.ed.gov/about/offices/list/ oii/nonpublic/nclbinfo.html

Nussbaum, M. C. (2015). Philosophy and economics in the capabilities approach: An essential dialogue. Journal of Human Development and Capabilities, 16(1), 1-14. https://doi.org/ 10.1080/19452829.2014.983890

OCDE. (2018). The resilience of students with an immigrant background: Factors that shape well-being, OECD reviews of migrant education. OECD Publishing.

Ogbu, J. (1982). Cultural discontinuities and schooling. Anthropology \& Education Quarterly, 13, 290-307. https://doi.org/10.1525/aeq.1982.13.4.05x1505w

Pardo, J. L. (2016) Estudios del malestar. Políticas de la autenticidad en las sociedades contemporáneas. Anagrama.

Paris, D. \& Alim, H. S. (2017). Culturally sustaining pedagogies: Teaching and learning for justice in a changing world. Teachers College Press.

Paris, D. \& Ball, A. (2009). Teacher knowledge in culturally and linguistically complex classrooms: Lessons from the golden age and beyond. En L. Morrow, R. Rueda, \& D. Lapp (Eds.), Handbook of research on literacy instruction: Issues of diversity, policy, and equity (pp. 379-395). Guilford.

Popkewitz, T. S. (2002). Infancia, modernidad y escolarización: Nacionalidad, Ciudadanía, Cosmopolitismo y «los otros» en la constitución del sistema educativo norteamericano. En M. A. Pereyra, J. C. González-Faraco, y J. M. Coronel (Comp.), Infancia y escolarización en la modernidad tardía (pp. 17-70). Akal.

Popkewitz, T. S. (2010). Inclusión y exclusión como gestos dobles en política y ciencias de la educación. Propuesta Educativa, 33, 11-28. https://www.redalyc.org/ pdf/4030/403041789003.pdf

Richards, H., Brown, A. \& Forde, T. (2006). Addressing diversity in schools: Culturally responsive pedagogy. Teaching exceptional children, 39(3), 64-68. https://doi. org/10.1177/004005990703900310

Rodríguez-Izquierdo, R. M. (2009). La investigación sobre educación intercultural en España. Education Policy Analysis Archives / Archivos Analiticos de Políticas Educativas, 17, 1-29. https://www.redalyc.org/articulo.oa?id=275019727004 
ROSA MARÍA RODRÍGUEZ-IZQUIERDO Y JUAN CARLOS GONZÁLEZ-FARACO

LA EDUCACIÓN CULTURALMENTE RELEVANTE: UN MODELO PEDAGÓGICO PARA LOS ESTUDIANTES

DE ORIGEN CULTURAL DIVERSO. CONCEPTO, POSIBILIDADES Y LIMITACIONES

Rodríguez-Izquierdo, R. M. (2010a). Éxito académico de los estudiantes inmigrantes. Factores de riesgo y de protección. Educación XX1, 13(1), 101-123. https://doi.org/10.5944/ educxx1.13.1.279

Rodríguez-Izquierdo, R. M. (2010b). Éxito y fracaso escolar de la primera y la segunda generación de estudiantes de origen inmigrante. ESE. Estudios sobre Educación, 19, 97-118. https://dadun.unav.edu/bitstream/10171/18327/2/ESE19_Articulo5.pdf

Rodríguez-Izquierdo, R. M. (2011a). Discontinuidad cultural: Estudiantes inmigrantes y éxito académico. Aula abierta, 39(1), 69-80. https://dialnet.unirioja.es/servlet/ articulo?codigo $=3353887$

Rodríguez-Izquierdo, R. M. (2011b). La enseñanza como práctica cultural: la gestión de aulas diversas. Enseñanza \& Teaching. Revista interuniversitaria de didáctica, 29(1), 53-70. https://dialnet.unirioja.es/servlet/articulo?codigo=3726723

Rodríguez-Izquierdo, R. M., González Falcón, I. \& Permisán, C. G. (2020). Teacher beliefs and approaches to linguistic diversity. Spanish as a second language in the inclusion of immigrant students. Teaching and Teacher Education, 90. https://doi.org/10.1016/j. tate.2020.103035

Santos Rego, M. A. y Lorenzo, M. (Eds.). (2012). Estudios de pedagogía intercultural. Octaedro.

Santos Rego, M. A., Lorenzo Moledo, M., Priegue Caamaño, D. y Torrado Quintela, J. (2020). Variaciones en los hábitos de estudio en función del género y origen étnico-cultural del alumnado y su relación con el rendimiento escolar. Revista Complutense de Educación, 31(2), 163-171. https://doi.org/10.5209/rced.62000

Sassen, S. (2010). Territorio, autoridad y derechos. Katz Editores.

Sen, A. (2011). Desarrollo y crisis global. Editorial Complutense.

Sleeter, C. (2008). An invitation to support diverse students through teacher education. Journal of Teacher Education, 59(3), 212-219. https://doi.org/10.1177/0022487108317019

Sleeter, C. (2011). The academic and social value of ethnic studies: A research review. National Education Association.

Sousa Santos, B. de (2006). Globalización y democracia. Archipiélago, 73-74, 111-125.

Suárez-Orozco, C., Suárez-Orozco, M. \& Todorova, I. (2008). Learning a new land: Immigrant students in American society. Harvard University Press.

Talavera Pérez, I y Guzmán Rosquete, R. (2018). La evaluación psicopedagógica del alumnado cultural y lingüísticamente diverso: un estudio exploratorio sobre las prácticas de los orientadores. Revista Complutense de Educación, 29(1), 11-26. https://doi.org/10.5209/ RCED. 51916

UNESCO. (2000). Marco de acción de Dakar: Educación para todos: Cumplir nuestros compromisos comunes (con los seis marcos de acción regionales). Foro Mundial sobre la Educación. UNESCO.

Valencia, R. (2010). Dismantling contemporary deficit thinking. Educational thought and practice. Taylor and Francis.

Vertovec, S. (2012). Diversity and the social imaginary. European Journal of Sociology, 53, 287-312. https://doi.org/10.1017/S000397561200015X

Villegas, A. M. y Lucas, T. (2002). Preparing culturally responsive teachers: Rethinking the curriculum. Journal of Teacher Education, 53(13), 20-32. https://doi. org/10.1177/0022487102053001003 
ROSA MARÍA RODRÍGUEZ-IZQUIERDO Y JUAN CARLOS GONZÁLEZ-FARACO LA EDUCACIÓN CULTURALMENTE RELEVANTE: UN MODELO PEDAGÓGICO PARA LOS ESTUDIANTES DE ORIGEN CULTURAL DIVERSO. CONCEPTO, POSIBILIDADES Y LIMITACIONES

Walsh, C. (2009). Interculturalidad, estado y sociedad. Luchas (de)coloniales de nuestra época. Ediciones Abya-Yala.

Walsh, C. (2010). Interculturalidad crítica y educación intercultural. En J. Viaña, L. Tapia, y C. Walsh, Construyendo interculturalidad crítica (pp. 75-96). Instituto Internacional de Integración-Convenio Andrés Bello (III-CAB). https://aulaintercultural.org/2010/12/14/ interculturalidad-critica-y-educacion-intercultural/ 